\title{
Study of structure - anti-HIV activity relationship of shilajit samples with a use of cell-based assays and solution state ${ }^{13} \mathrm{C}$ NMR spectroscopy
}

Konstantinov A.I. ${ }^{1}$, Zhernov Yu.V. ${ }^{2}$, Perminova I.V. ${ }^{1}$

${ }_{1}^{1}$ Lomonosov MSU, Department of Chemistry, Moscow, Russia, konstant@med.chem.msu.ru

${ }^{2} \mathrm{NRC}$ Institute of Immunology FMBA of Russia, Moscow

doi: 10.36291/HIT.2019.konstantinov.091

Shilajit that emerges as a resinous exudation from rocks of mountains is considered to be a complex product of biotransformation of plant residues [1]. Antiviral activity of shilajit resulting from its biological activity was repeatedly reported [1-2]. However, structural composition of its active components remains unknown. In this study we investigated inhibitory effect of dried water extractions of 11 raw shilajit samples originated from different geographic regions on HIV in cell-based assays. Structural analysis of the samples were conducted using quantitative solution state ${ }^{13} \mathrm{C}$ NMR spectroscopy. The HIV-1 899A was used in this study to infect TZM-bl cells (NIH AIDS Reagent Program No. 8129, USA) obtained by genetic engineering of HeLa cells. EC $_{50}$ vallues of dried shilajit water extractions were calculated using the Reed and Muench method. To obtain structural composition data, recorded ${ }^{13} \mathrm{C}$ NMR spectra of shilajit samples were divided into intervals corresponding to typical structural units of natural organic matter (Figure 1) and integrated.

A strong direct correlation $(R=0.82 ; p$-value $=0.02)$ between $E_{50}$ values measured for shilajit samples and percentages of non-phenolic aromatic carbon was observed. Thus, aromatic structures were found to reduce antiviral activity of shilajit, and this was drastically different from the results obtained later for humic substances [3].

Antiviral activity of shilajit samples indicates that these substances hold significant promise as safe and efficacious antiviral drugs for treatment of HIV infection. Further studies of their antiviral activity and identification of antiviral structural components should include preliminary sample fractionation and separation of low-molecular aromatic compounds.

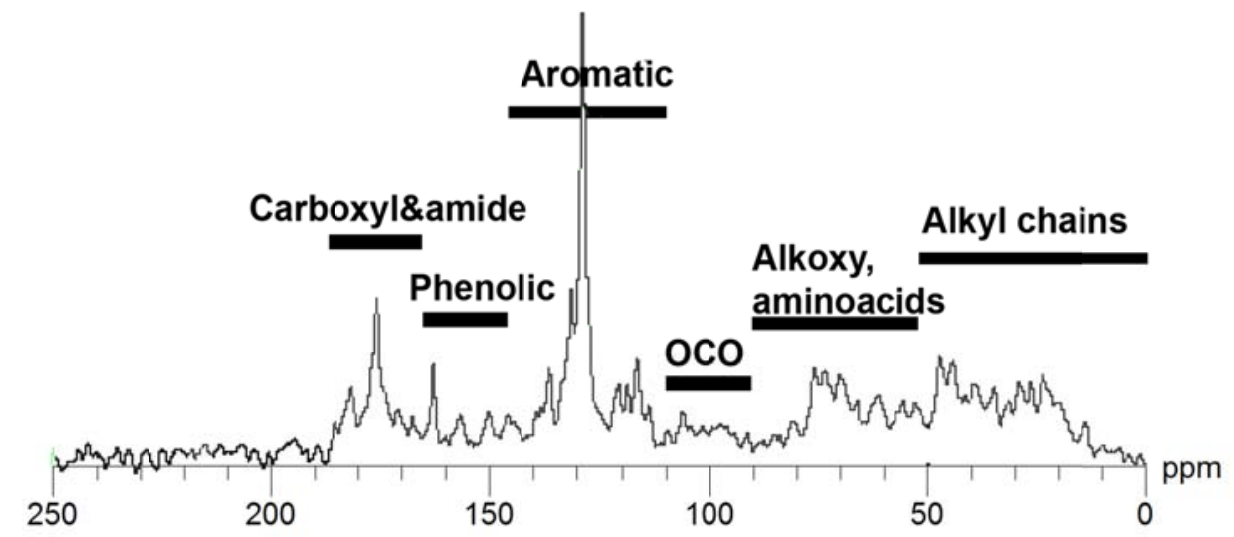

Figure 1. A typical ${ }^{13} \mathrm{C}$ NMR spectrum of shilajit in $\mathrm{D}_{2} \mathrm{O}$ and regions of typical structures.

Acknowledgements. This study was supported by the Russian Foundation for Basic Research (grant 16-03-01057 A). We thank Dr. M.I. Savinykh ("Sibdalmumiyo" Ltd, Novokuznetsk) for his help in raw shilajit sample collection.

References

1. Agarwal S.P. et al. // Phytother. Res. 2007. 21:401-405.

2. Cagno V. et al. // J. Ethnopharmacol. 2015. 166:129-134.

3. Zhernov Yu.V. et al. // New J. Chem. 2017. 41:212-224. 